\title{
Compare of analytic and numerical solution of parametric oscillator with perturbation and its analytical solution
}

\author{
Pavol Oršanský1,* and Branislav Ftorek ${ }^{1}$ \\ ${ }^{1}$ Department of Applied Mathematics, Faculty of Mechanical Engineering, University of Žilina, \\ Univerzitná 1, 01026 Žilina, Slovakia
}

\begin{abstract}
In this paper we deal with an approximative analytical solution of a second order differential equation with a fast time periodic modulation. We use for this type of the equation a homotopy perturbation method. The validity of this approximative solution is verified by numerical experiments, where we achieve considerable consistency of doth soliutions.
\end{abstract}

Keywords: oscillations, asymptotic solution, Mathieu equation, Homotopy perturbation method

\section{Introduction}

Most of engineering problems, especially oscillation equations are nonlinear or the equations have non-constant parameters. It is difficult to solve such equations, especially analytically. Time-periodic modulation of system parameters may lead to resonances, instabilities and some interesting physical phenomena. The simplest system exhibiting parametric resonances is the harmonic oscillator with a frequency that varies periodically with time. The equation of motion of a simple harmonic oscillator whose frequency is modulated by a time-dependent zero-average perturbation $\xi(t)$ is

$$
\ddot{x}+\omega_{0}^{2}[1+\xi(t)] x=0,
$$

where $x$ is the displacement and $\omega_{0}$ is the natural frequency of the oscillator. The equation (1) has a fixed point at $x=0$ regardless of the type of modulation. When the modulation is absent, the fixed point is neutrally stable. That is, near $x=0$ the displacement neither grows without bound nor decays. However, in the modulation case the fixed point may be unstable for certain temporal modulations. An example of unstable mode of oscillation system with its phase portrait can be seen in Fig. 1. If the modulation is specified to be cosinusoidal, the equation (1) is called the Mathieu equation:

$$
\ddot{x}+\left[\omega_{0}^{2}-\varepsilon \cos t\right] x=0 .
$$

\footnotetext{
* Corresponding author: pavol.orsansky@,fstroj.uniza.sk

Reviewers: Vladimír Dekýš, Zbigniew Kłos
} 

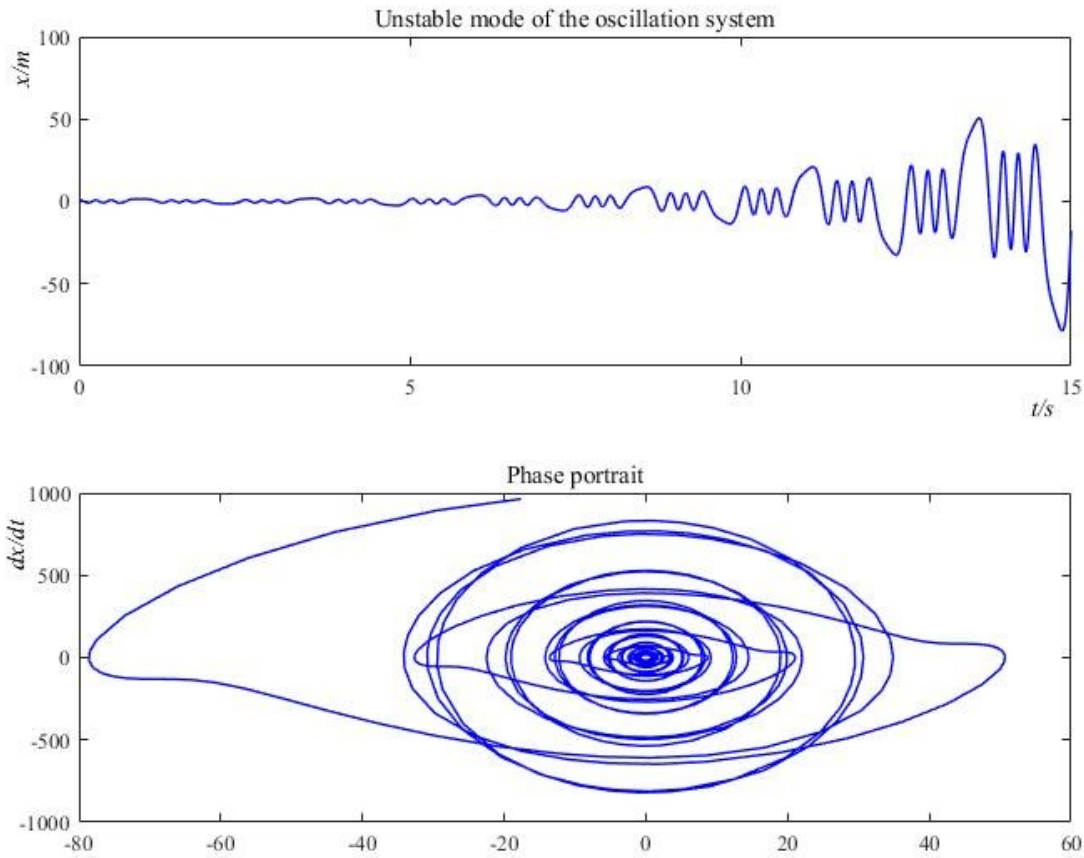

Fig. 1. Unstable mode of oscillation system with its phase portrait : $\ddot{x}+\omega_{0}^{2}[1+\sin B t]=0$, where $\omega_{0}=20, B=\frac{\omega_{0}}{4}$

The solution of the Mathieu equation or the solution of some nonlinear equation may be find by using some general asymptotic methods. Many interesting physical phenomena are described by general differential equations with nonlinear or time dependent terms:

$$
\ddot{x}+\omega_{0}^{2} x+\varepsilon f(t, x, \dot{x})=0,
$$

where $f(t, x, \dot{x})$ is a real function and $\varepsilon$ is a small parameter. Perturbation technique is one the well-known methods [1,2]. If $\varepsilon=0$ then the solution of the previous equation has a form

$$
x(t)=A \sin \left(\omega_{0} t+\varphi\right),
$$

where the amplitude $A$ and the phase $\varphi$ are constants. We suppose the perturbation solution for $\varepsilon \neq 0$ in the form of an asymptotic series [3]:

$$
x(t)=A(t) \sin \left(\omega_{0} t+\varphi(t)\right)+\varepsilon \phi_{1}(t)+\varepsilon^{2} \phi_{2}(t)+\cdots+\varepsilon^{n} \phi_{n}(t)+\cdots,
$$

where $A(t), \varphi(t), \phi_{1}(t), \ldots, \phi_{n}(t), \ldots$ are unknown functions. However, the perturbation methods are not useful for strongly nonlinear equations [2]. Another asymptotic method is well known as a WKB-method, or Wentzel-Kramers-Brillouin method (see [4]) for the equation with a large parameter $\lambda$, that is, $\lambda \rightarrow \infty$

$$
\ddot{x}+f(x, \lambda) x=0 .
$$




\section{Homotopy perturbation method (HPM)}

In the paper [5] we found an asymptotic solution of the initial values problem for second order differential equation with a friction term and with a fast time periodic modulation:

$$
\begin{gathered}
\ddot{x}+2 \gamma \dot{x}+\omega_{0}^{2}[1+\lambda \sin B t] x=0, \\
x(0)=1,
\end{gathered}
$$

where $\lambda>0, \gamma>0, \omega_{0}>0$, are parameters of the oscillation system.

An approximative solution:

$$
\begin{gathered}
x(t)=e^{-\gamma t} \cos (\Omega t)[1+\varepsilon \sin B t], \\
\Omega=\sqrt{\omega_{0}^{2}-\gamma^{2}+\frac{\varepsilon \lambda \omega_{0}^{2}}{2},} \\
\varepsilon=\frac{-B^{2}}{\lambda \omega_{0}^{2}}+\sqrt{\frac{B^{4}}{\lambda^{2} \omega_{0}^{4}}+2,}
\end{gathered}
$$

for a fast time periodic modulation (the parameter $\varepsilon$ is sufficiently small) was obtained.

Now we use for this type of the problem and for large scale of parameters a homotopy perturbation method [2]. HPM can solve a large class of nonlinear problems with approximations converging rapidly to accurate solutions. Let us consider the nonlinear equation:

$$
A(u)-f(r)=0, \quad r \in \Omega,
$$

with some boundary condition:

$$
B\left(u, \frac{\partial u}{\partial n}\right)=0, \quad r \in \partial \Omega,
$$

where $A$ is a nonlinear differential operator with decomposition:

$$
A=L+N \text {, }
$$

$L$ and $N$ are the linear and nonlinear parts of $A, f(r)$ is a known analytical function, $\partial \Omega$ is the boundary solution domain $\Omega$ and $\frac{\partial u}{\partial n}$ denotes differentiation along the outwards normal to $\partial \Omega$. To solve (10) we construct a homotopy:

$$
H(v, p)=(1-p)\left[L(v)-L\left(u_{0}\right)\right]+p[A(v)-f(r)]=0, \quad p \in[0,1],
$$

where $v(r, p): \Omega \times[0,1] \rightarrow \mathbb{R}, p$ is an embedding parameter and $u_{0}$ is the first approximation that satisfies the boundary condition. In this method $v(r, p)$ can be expanded into a power series with parameter $p$, as the following:

$$
v(r, p)=v_{0}+p v_{1}+p^{2} v_{2}+p^{3} v_{3}+\cdots
$$

The best approximation for the solution we get by setting $p=1$ :

$$
u=\lim _{p \rightarrow 1} v=v_{0}+v_{1}+v_{2}+v_{3}+\cdots .
$$




\section{Solution using HPM}

Now we will use the basic idea from the previous section and from the paper [2].

We consider the equation of motion of a damped harmonic oscillator whose frequency is modulated by a time-dependent zero-average perturbation:

$$
\ddot{x}+2 \gamma \dot{x}+\left[\omega_{0}^{2}+\epsilon \sin B t\right] x=0,
$$

with initial conditions (for simplicity):

$$
x(0)=1, \frac{d x(0)}{d t}=-\gamma .
$$

A more convenient form of (12) for study is obtained on setting:

$$
x(t)=y(t) e^{-\gamma t} .
$$

We have:

$$
\ddot{y}+\left[\lambda^{2}+\epsilon \sin B t\right] y=0,
$$

where $\lambda^{2}=\omega_{0}^{2}-\gamma^{2}$. The homotopy (11a) has a form:

$$
H(v, p)=(1-p)\left[\ddot{u}+\lambda^{2} u\right]+p\left[\ddot{u}+\lambda^{2} u+\epsilon \sin B t u\right]=0, \quad p \in[0,1] .
$$

Now we assume the solution:

$$
u=v_{0}+p v_{1}
$$

and

$$
\dot{u}=\frac{d v_{0}}{d t}+p \frac{d v_{1}}{d t}, \quad \ddot{u}=\frac{d^{2} v_{0}}{d t^{2}}+p \frac{d^{2} v_{1}}{d t^{2}} .
$$

Substituting the previous relations in (16) and comparing coefficients of the terms with the identical powers of $p$, lead to:

$$
\begin{aligned}
& p^{0}: \frac{d^{2} v_{0}}{d t^{2}}+\lambda^{2} v_{0}=0, \\
& p^{1}: \frac{d^{2} v_{1}}{d t^{2}}+\lambda^{2} v_{1}=-\epsilon v_{0} \sin B t
\end{aligned}
$$

with initial conditions $v_{0}(0)=1, \frac{d v_{0}(0)}{d t}=0$ and $v_{1}(0)=0, \frac{d v_{1}(0)}{d t}=0$ (see (13) and (14)). The problem (18) has a solution:

$$
v_{0}=\cos \lambda t
$$

If we denote $\alpha=1-\frac{4 \lambda^{2}}{B^{2}}$, then the solution of (19) has two parts, general and particular solutions:

$$
\begin{gathered}
v_{1}=\frac{-\epsilon}{2 B \lambda}\left(1+\frac{1}{\alpha}\right) \sin \lambda t+\frac{\epsilon}{2 B \lambda} \cos \lambda t\left(\frac{-1}{\alpha} \cos B t \sin 2 \lambda t+\frac{2 \lambda}{B \alpha} \sin B t \cos 2 \lambda t\right)+ \\
+\frac{\epsilon}{2 B \lambda} \sin \lambda t\left(\cos B t+\frac{1}{\alpha} \cos B t \cos 2 \lambda t+\frac{2 \lambda}{B \alpha} \sin B t \sin 2 \lambda t\right) .
\end{gathered}
$$

With regard to (11b), (11c) and (14), (16), (17), the approximative solution has an exact form:

$$
x(t)=e^{-\gamma t}\left(v_{0}+v_{1}\right),
$$

where the unknown functions $v_{0}$ and $v_{1}$ are determinated by relations (20) and (21). 
From the relation (21) follow conditions of applicability of the main approximation method. It is desirable that $\frac{\epsilon}{2 B \lambda}$ is sufficiently small, that is, $\frac{\epsilon}{2 B \lambda} \rightarrow 0^{+}$and $\alpha=1-\frac{4 \lambda^{2}}{B^{2}} \neq 0$. The validity of this approximative solution is verified by numerical experiments. We obtained numerical results by the MATLAB environment. The comparison between analytical solutions and numerical solutions for the large scale of parameters are shown in the graphs, where numerical results represent blue dot-dashes lines and analytical results the red line (see Figures 2-5).

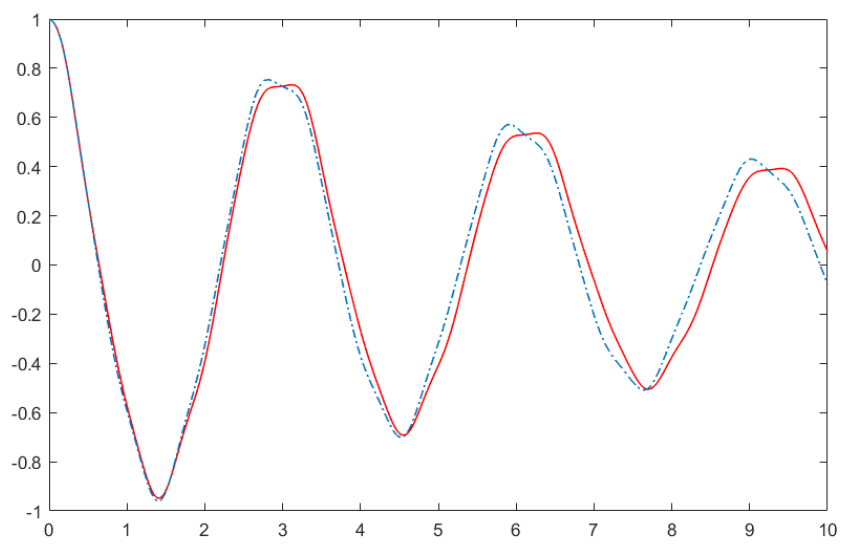

Fig. 2. Comparison between numerical (blue) and analytical (red) solution for parameters: $\gamma=0.1, \omega_{0}=2, \varepsilon=5, B=10$

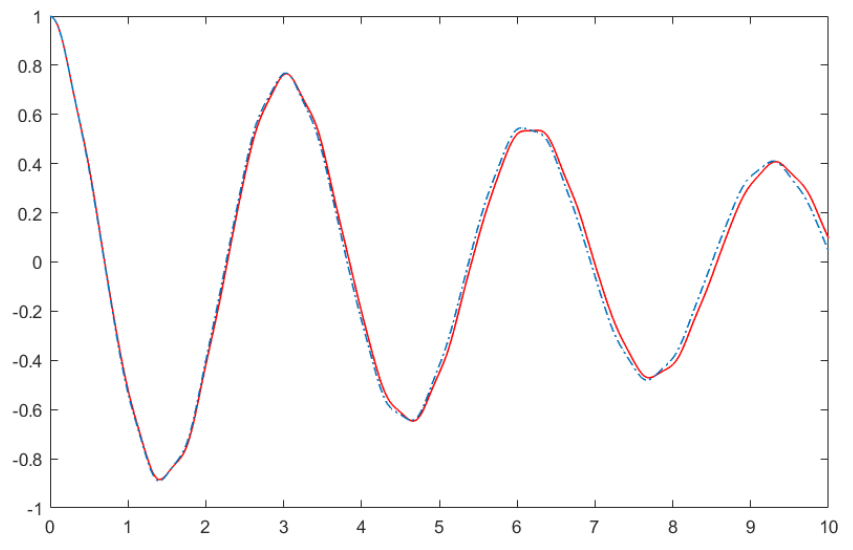

Fig. 3. Comparison between numerical (blue) and analytical (red) solution for parameters: $\gamma=0.1, \omega_{0}=2, \varepsilon=5, B=15$ 


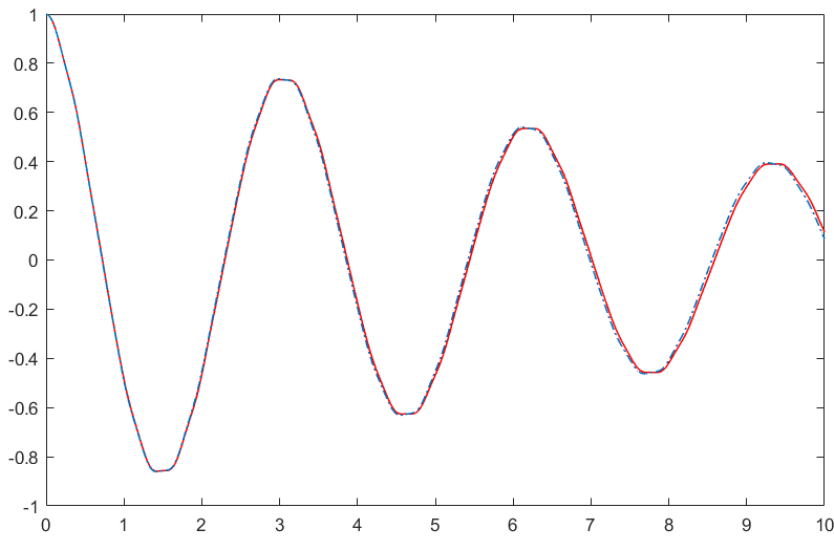

Fig. 4. Comparison between numerical (blue) and analytical (red) solution for parameters: $\gamma=0.1, \omega_{0}=2, \varepsilon=5, B=20$

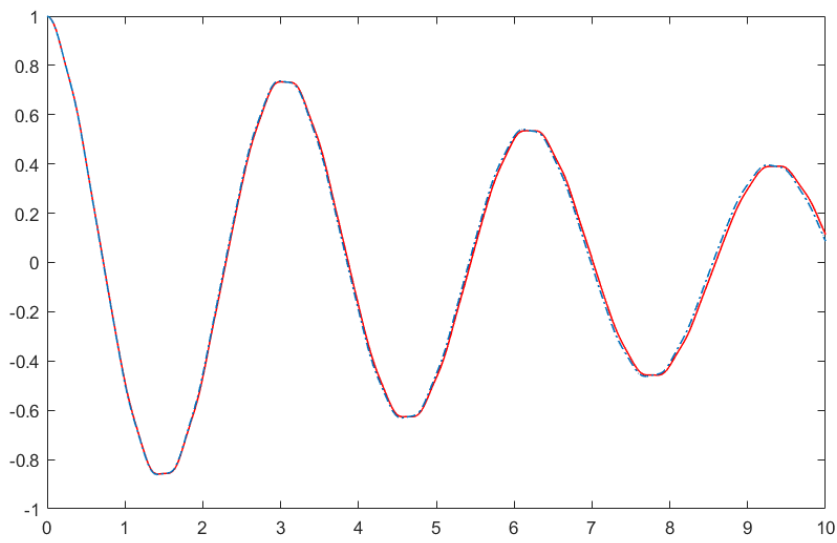

Fig. 5. Comparison between numerical (blue) and analytical (red) solution for parameters: $\gamma=0.1, \omega_{0}=2, \varepsilon=50, B=200$

\section{Conclusion}

The equation of motion (12) of a damped harmonic oscillator whose frequency is modulated by a time-dependent zero-average perturbation was investigated, as well as in papers [6-8]. Using the Homotopy perturbation method an approximative solution was obtained. This obtained asymptotic solution was compared with the numerical solution gained by the RungeKutta method. We have achieved quite a good concordance of both solutions for required small parameters $\left(\frac{\epsilon}{2 B \lambda} \rightarrow 0^{+}\right)$. Of course we must take into account the fact, that the numerical solution loaded with an error of the Runge-Kutta method. Also we should say that ours analytical solution is only quasi analytical, because the Homotopy perturbation method solve the nonlinear problem with approximations converging rapidly to accurate solutions, so it is only an approximation of the accurate solution. 
This work was supported by KEGA under the Grant No. 015ŽU-4/2017 Digital technologies for machine building study programs as a part of strategy "Internet of Things".

\section{References}

1. S. H. Strogatz: Nonlinear dynamics and chaos. Westview Press, Cambridge, (1994)

2. M. Bayat, I. Pakar, G. Domairry: Recent developments of some asymptotic methods and theit applications for nonlinear vibration equations in engineering problems: A review. Latin American Journal of Solids and Structures 1, 1-93, (2012)

3. R. A. Struble: Nonlinear differential equations. McGraw-Hill book company, New York, (1962)

4. J. D. Murray: Asymptotic analysis. Applied mathematical sciences, Springer-Verlag, New-York, (1984)

5. B. Ftorek, P. Oršanský, H. Šamajová: Asymptotic case of the parametric oscillations. Dymamesi 2016: international colloquium, Prague: Institute of Thermomechanics AS CR, (2016)

6. R. Kawai, K. Lindenberg, C. Van den Broeck: Parametrically modulated oscillator dimer: an analytic solution. Physica A, 312, 119-140, (2002)

7. J. Zapoměl, V. Dekýš, P. Ferfecki, A. Sapietová, M. Sága, M. Žmindák: Identification of Material Damping of a Carbon Composite Bar and Study of Its Effect on Attenuation of Its Transient Lateral Vibrations. International Journal of Applied Mechanics, 7, 1-18, (2015)

8. E. Wiszt, E. Wisztová: An approximative solution of some second order differential equation with variable coefficients. Studies of the University of Žilina, Math. Series, 21, 45-52 (2007) 